\title{
Medical Image of the Week: Pneumothorax with Air Bronchograms
}

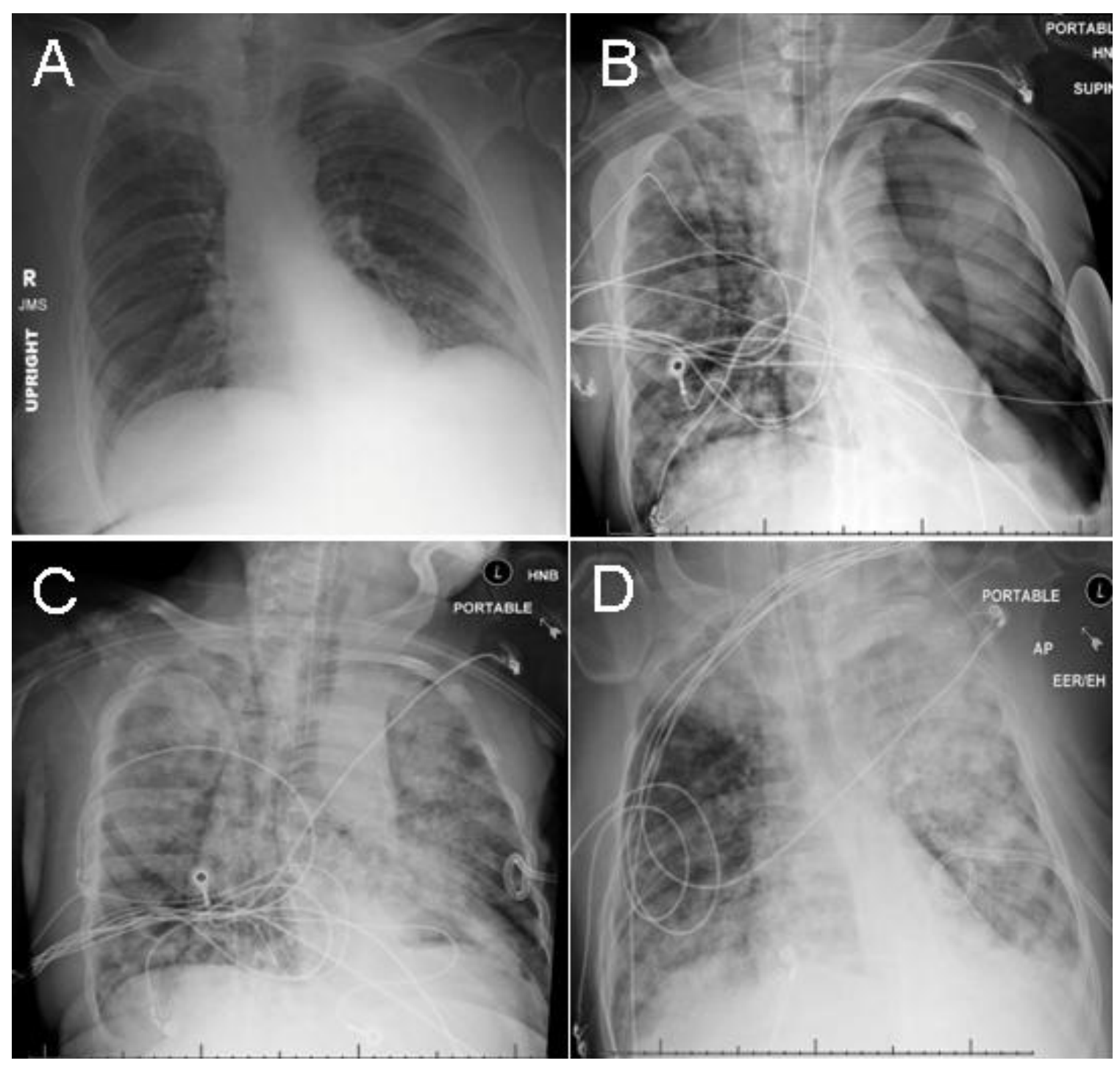

Figure 1. Panel (A) shows mild congestion with prominent bronchovascular markings. Panel (B) shows a large left pneumothorax with total collapse of the left lung marked by extensive airspace opacities and distinct air bronchograms. Panel (C) shows interval placement of a left-sided pigtail catheter with partial resolution of the left pneumothorax. There is persistent collapse of the medial aspect of the left upper lobe. Panel (D) shows complete resolution of the left pneumothorax and left lung atelectasis with continued bilateral airspace disease.

Development of pneumothoraces in critically ill patients is commonly encountered in the critical care unit (ICU). Incidence has been reported between $4-15 \%$ of patients. In most instances, pneumothorax in the ICU is considered a medical emergency especially when the patient is mechanically ventilated (1). Here, we present a 61-year-old man with a past medical history of insulin 
dependent diabetes and paraplegia from prior spine injury who presented with acute respiratory distress after a pulseless electrical activity cardiac arrest. Cardiopulmonary resuscitation (CPR) was initiated by emergency medical services at home, and continued and the emergency department (ED) for a total of 30 minutes. The patient presented previously to the ED, one week prior, for a mild respiratory illness and tested positive for influenza B. He was sent home on oseltamivir. His family is unsure of compliance with medication but reported he was clinically stable up to the morning of presentation. The patient, as shown in the images, developed a left pneumothorax complicating an "adult respiratory distress syndrome (ARDS)- like" picture probably due to positive pressure ventilation with high positive end expiratory pressure, CPR, or both. The patient underwent immediate chest tube placement and with successful lung reexpansion. Unfortunately, his hemodynamic status/septic shock/multi-organ system failure continued to deteriorate within hours and he expired despite maximal support. Pneumothorax in patients with ARDS has higher morbidity and mortality compared to other critically ill patients due to the high-pressure needed during mechanical ventilation. This places patients at a high risk for the rapid progression to tension pneumothorax and even death. Therefore, in this high-risk population, a pneumothorax requires a high index of suspicion, prompt recognition, and immediate intervention (2).

Huthayfa Ateeli, MBBS and Steve Knoper, MD.

Department of Medicine, Division of Pulmonary, Critical Care, Sleep and Allergy Medicine. University of Arizona, Tucson, AZ USA

\section{References}

1. Yarmus L, Feller-Kopman D. Pneumothorax in the critically ill patient. Chest. 2012 Apr;141(4):1098-105. [CrossRef] [PubMed]

2. Gattinoni L, Bombino M, Pelosi P, Lissoni A, Pesenti A, Fumagalli R, Tagliabue $M$. Lung structure and function in different stages of severe adult respiratory distress syndrome. JAMA. 1994 Jun 8;271(22):1772-9. [CrossRef] [PubMed] 